\title{
eJRIEPS
}

Ejournal de la recherche sur l'intervention en éducation physique et sport

$10 \mid 2006$

Varia

\section{Actualités des thèses}

\section{OpenEdition}

\section{Journals}

Édition électronique

URL : https://journals.openedition.org/ejrieps/7479

DOI : 10.4000 /ejrieps.7479

ISSN : 2105-0821

Éditeur

ELLIADD

Édition imprimée

Pagination : 157-163

\section{Référence électronique}

«Actualités des thèses », eJRIEPS [En ligne], 10 | 2006, mis en ligne le 01 juillet 2006, consulté le 29 octobre 2021. URL : http://journals.openedition.org/ejrieps/7479; DOI : https://doi.org/10.4000/ ejrieps.7479

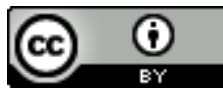

La revue eJRIEPS est mise à disposition selon les termes de la Creative Commons Attribution 4.0 International License. 


\section{Actualités des thèses.}

Sigolène COUCHOT-SCHIEX. Contributions aux effets du genre de l'enseignant en EPS. Etude descriptive dans trois APSA : gymnastique, badminton, handball. Amade-Escot Chantal, Professeure en Sciences de l'Education, Université de Toulouse ; Anaut MariP, Professeure en Sciences de l'Education, Université Lyon 2 ; Cogérino Geneviève, professeure en STAPS, Université d'Amiens ; Develay Michel, Professeur en Science de l'Education, Université Lyon 2 (directeur); Terret Thierry, Professeur en STAPS, Université Lyon 1

Université Lumière Lyon 2. Sciences de l'Education

Thèse soutenue le : 9 décembre 2005.

Cette étude s'appuie sur 85 vidéos tournées avec l'accord de 16 enseignants d'EPS de collège au cours de leçons et d'évaluation en gymnastique, badminton et handball. Ces hommes et ces femmes sont répartis en quatre groupes de genre (masculin, androgyne, non différencié et féminin). Nous cherchons à partir d'une analyse de contenu à décrire si des effets de genre se font jour à travers des indicateurs variés portant aussi bien sur les contenus d'enseignement et les évaluations proposées que sur les interactions entre l'enseignant et les élèves différenciés selon leur sexe. Une étude quantitative quant au nombre et à la durée des interactions est également effectuée. Au-delà des effets du genre et du sexe de l'enseignant en EPS, cette discipline est questionnée dans ses pratiques notamment au regard de la mixité et de l'égalité des sexes en rapport avec les pratiques sportives auxquelles elle fait référence.

Mots clés: genre, EPS, enseignant, contenus, interactions, mixité, pratiques sportives

Anne-Laure DEMIAZ-DIAZ. L'élève transparent: une étude clinique de la rencontre à l'école par l'analyse de discours d'enseignants

BIRMES Philippe, Professeur de Psychiatrie, Université Paul Sabatier, Toulouse III ; BLANCHARD-LAVILLE Claudine, Professeur en Sciences de l'Education, Université Paris X (Rapporteur) ; CLANCHE Pierre, Professeur en Sciences de l'Education, Université Bordeaux II (Rapporteur) ; SAURET Marie-Jean, Professeur en Psychologie Clinique et Psychopathologie, Université Toulouse III. ; TERRISSE André, Professeur en Sciences de l'Education, IUFM Toulouse (Directeur de Thèse).

Université Paul Sabatier 
Thèse soutenue le 10 novembre 2005.

La présente étude s'attache à interroger l'existence d'élèves « adjectivés » transparents dans une classe. Ces élèves sont sources d'oublis par les enseignants. « J'avais du mal à me souvenir de leurs prénoms, de leurs noms et de leurs existences " déclare une enseignante. Trois enseignants ont été rencontrés pour cinq entretiens chacun. JeanLouis est professeur des écoles, Irène, professeur d'E.P.S., et Béatrice, enseignante d'anglais. Une analyse des discours en trois temps (analyse narrative, analyse de la causalité de la transparence et analyse de l'énonciation) s'attache à repérer les motifs de ces méprises, erreurs, oublis. Partant de la psychanalyse, deux constructions sont proposées : l'élève-miroir et l'enseignant «castré » permettent de rendre compte de la transparence. Et la question de la pulsion scopique est mise à l'œuvre pour en donner un sens. L'inquiétante étrangeté de l'élève transparent ferait de ce dernier l'objet présentifiant la jouissance de l'enseignant. La transparence serait alors le voile mis par l'enseignant face à l'irruption d'un réel dans la rencontre à l'élève transparent. Ne pas voir l'élève serait la solution trouvée par l'enseignant pour n'en rien vouloir savoir (ça-voir). Cette étude s'inscrit dans une lignée de travaux déjà existants, ceux qui privilégient l'étude de cas et qui questionnent l'inconscient dans la classe. Cependant, la rencontre à l'Ecole visitée de la sorte argumente pour la dimension de l'inconscient, c'était oublié, et en faveur du Réel, ce point où entre la cause et ce qu'elle affecte il y a toujours clocherie. »

Mots-clés: Elève transparent, Rencontre à l'Ecole, Discours d'enseignants, Etude de cas et construction, Psychanalyse, Inconscient.

Isabelle JOURDAN. L'évolution du rapport au savoir comme "révélateur" des logiques de professionnalisation: études de cas en formation initiale en EPS à I'IUFM de MidiPyrénées.

ALTET Marguerite, Professeure en Sciences de l'Education, Université de Caen; CAILLOT Michel, Professeur en Sciences de l'Education, Université Paris V La Sorbonne ; DAVID Bernard, Professeur en STAPS, Université de Créteil ; PERRENOUD Philippe, Professeur en Sciences de l'Education, Université de Genève; TERRISSE André, Professeur en Sciences de l'Education, IUFM Midi-Pyrénées Université Paul Sabatier

Thèse soutenue le 16 juin 2005. 
Dans le contexte de la formation initiale en éducation physique et sportive (EPS), notre recherche a pour objet d'établir un lien entre les logiques de professionnalisation et le rapport au savoir des étudiants en première année d'IUFM (PLC1) et des professeurs stagiaires en deuxième année d'IUFM (PLC2) en éducation physique et sportive (EPS). Nous faisons le choix de privilégier la singularité et le sens que prend le savoir pour le sujet (Charlot, 1997 ; Beillerot et al, 1996). La spécificité de la discipline EPS, la place du corps, l'ancrage praxéologique de ces savoirs, nous conduit à étudier plus spécifiquement son rapport aux Activités Physiques, Sportives et Artistiques (APSA). Nous nous centrons sur la problématique de la professionnalisation (Altet, 2000, Bourdoncle, 1991, Lang, 1999), devenue un enjeu de la formation initiale et nous analysons les différentes logiques de professionnalisation à travers la notion de rapport au savoir. Nous estimons que les rapports au savoir sont un révélateur des logiques de professionnalisation déclarées. Nous nous inscrivons dans une démarche qualitative, de type exploratoire et longitudinal. Suivant une démarche clinique d'étude de cas, nous avons réalisé auprès de trois étudiants et à trois professeurs stagiaires en EPS, deux entretiens semi-directifs de recherche à un an d'intervalle. Nous avons pu mettre en évidence trois logiques de professionnalisation différenciées d'étudiants et de professeurs stagiaires selon trois idéaux-types de rapport au savoir.

Mots clés: rapport au savoir, rapport aux APSA, logique de professionnalisation, formation initiale, EPS, acte d'enseignement / apprentissage, étude de cas, recherche clinique. 